\title{
Multi-coated magnetoelectroelastic composites with functionally graded interphases
}

\author{
A. Bakkali ${ }^{1}$, L. Azrar ${ }^{1,2}$ and A. A. Aljinaidi ${ }^{2}$ \\ ${ }^{1}$ Faculty of Sciences and Techniques of Tangier, Abdelmalek Essaâdi University; Tangier; Morocco \\ ${ }^{2}$ Department of Mechanical Engineering, Faculty of Engineering, King Abdulaziz University, Jeddah, Saudi Arabia
}

\begin{abstract}
The aim of this work is to develop a micromechanical modeling to predict the effective properties of multi-coated magnetoelectroelastic composites with functionally graded interphases. The localization equations are derived based on the integral equation and on the interfacial operators. Magnetoelectroelastic composites with functionally graded interphases are analyzed and the effective properties are obtained. Based on different micromechanical models, the effects of the volume fractions, shapes of the multi-coated inclusions, thickness of the coatings and graded interphase on the effective properties are deeply analyzed.
\end{abstract}

Keywords: Multi-coated inclusion, functionally graded interphase, magnetoelectroelastic, micromechanical, Mori-Tanaka, Self Consistent, Incremental Self Consistent.

\section{Introduction}

Magnetoelectroelastic composites have attracted the attention of many researchers and engineers due to their attractive properties and to their applications in aerospace, automotives and medical imagery. One of the properties that make these composites very special is the magnetoelectric effect. The magnetoelectric effect, does not exist in any phase, is induced through the interaction between phases.

The effective properties of magnetoelectroelastic composites can be deeply influenced by the properties of the interphase between constituents. So, for accurate prediction of the effective properties of the magnetoelectroelastic composites, the nature of the interphase has to be taken into account in the modeling. The interphase may have continuous properties as it can have functionally graded properties. Functionally graded interphase refers to an interphase with properties that have spatially varying characteristics from a phase to another.

Various micromechanical models have been elaborated for piezoelectric composites, [1], as well as for magnetoelectroelastic composites. [2] investigated the effective properties of composite consisting of piezomagnetic inhomogeneities embedded in a nonpiezomagnetic matrix by using a unified energy method and the Mori-Tanaka and Dilute approaches. [3] extended the micromechanical Self Consistent, Mori-Tanaka and Dilute to study the coupled magnetoelectroelastic composite materials. [4] developed a mean field MoriTanaka model to calculate the effective magnetoelectroelastic moduli of matrix-based multiferroic composites by emphasizing the effects of shape and orientation distribution of second phase particles composites.

[5] developed a finite element analysis and micromechanics based averaging of a representative volume element to determine the effective dielectric, magnetic, mechanical, and coupled-field properties of an elastic matrix reinforced with piezoelectric and piezomagnetic fibers. The effective properties of multiphase magnetoelectroelastic materials have been predicted by [6] based on various micromechanical models. In this paper, the coated inclusion concept for magnetoelectroelastic materials has been elaborated. This concept has been used by [7, 8] for the effective properties of elastic composites and multi-coated ellipsoidal elastic inclusions and eigenstrains. As the coating and particularly the functionally graded interphase may have a big influence on the effective properties, this research work is dedicated to the modeling and investigation of these interphase effects. Various micromechanical modelings and predictions of effective properties of multi-coated magnetoelectroelastic composites with functionally graded interphases are elaborated.

\section{Mathematical formulation}

\subsection{Multi-coated composites}

Let us consider a multi-coated magnetoelectroelastic composite as presented in figure1. The topology of this magnetoelectroelastic multi-coated inclusion problem is described by an inclusion of a volume $\mathrm{V}_{1}$ whose behavior 
is described by the magnetoelectroelastic moduli $E^{1}$ surrounded by (n-1) thin coatings of other materials whose behaviors are described by their respective magnetoelectroelastic moduli $E^{i}$ and their volumes $V_{i}$ with $i \in\{2,3, \ldots . n\}$. The multi-coated inclusion is embedded in a homogenous media called the matrix whose behavior is described by the magnetoelectroelastic moduli $E^{M}$. In this analysis, the interphases are assumed perfectly bonded.

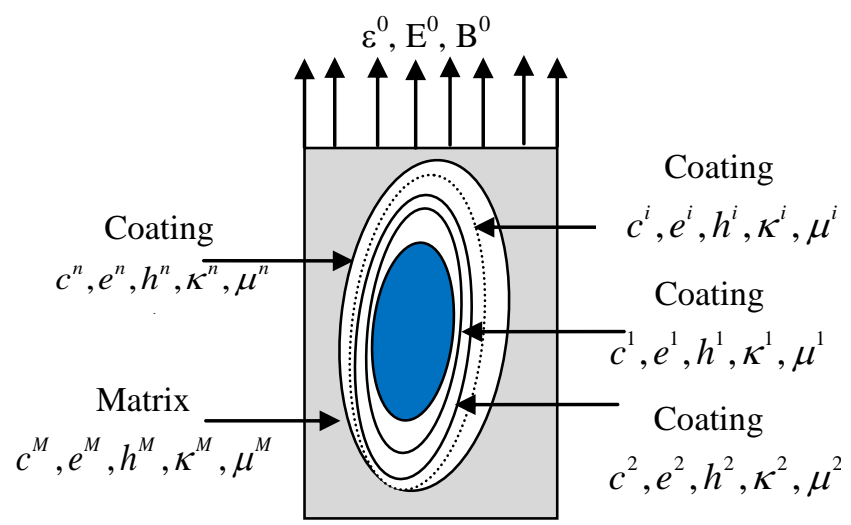

Fig. 1. Topology of the multi-coated inclusion. $\varepsilon^{0}, E^{0}$ and $B^{0}$ represent the macroscopically applied fields.

The integral equation linking the local generalized strain field $Z_{K l}(r)$ with the strain field of the reference medium $Z^{0}{ }_{K l}$ is given by the following integral equation (see [6] for more details):

$$
Z_{K l}(r)=Z_{K l}^{0}(r)-\sum_{k=0}^{n} \int_{V_{k}} \Gamma_{i J K l}\left(r-r^{\prime}\right) \Delta E_{i J M n}^{(k / 0)} Z_{M n}\left(r^{\prime}\right) d V^{\prime}(1)
$$

where $\mathrm{V}_{\mathrm{k}}$ the volume of the phase $\mathrm{k}, \Gamma_{i J K l}=-G_{J K, i l}$ is the condensed expression of nine Green's tensor [1].

To solve the integral equation (1) $Z_{M n}\left(r^{\prime}\right)$ is replaced by its average over the multi-coated inclusion volume $Z_{M n}\left(r^{\prime}\right)=\sum_{k=1}^{n} Z_{M n}^{k} \theta^{k}(r)$ in which $\theta^{k}(r)$ equals 1 inside $\mathrm{V}_{\mathrm{k}}$ and 0 outside. Based on some mathematical developments, the integrals equation (1) is transformed to the following localization equation.

$\sum_{k=1}^{n} \frac{V_{k}}{V_{I}}\left(I_{K l M n}+\frac{1}{V_{k}} T_{i J K l}^{I k}\left(E^{0}\right) \Delta E_{i J M n}^{(k / 0)}\right) Z_{M n}^{k}=Z_{K l}^{0}$

To complete the localization step, the interfacial operators are introduced which give the generalized strain jump field across an interface between two different phases by the following equation:

$Z_{M n}^{k+1}(r)-Z_{M n}^{k}(r)=P_{i J M n}^{k+1}\left(E^{K+1}, N\right)\left(E_{i J R s}^{k}-E_{i J R s}^{k+1}\right) Z_{R s}^{k}(r)(3)$

where $P_{i J M n}^{k+1}$ is the magnetoelectroelastic interfacial operator of the phase $(\mathrm{k}+1)$ which depends only on the property of the material phase $(\mathrm{k}+1)$ and on the unite normal N.
The use of equations (2) and (3) leads to the derivation of the expression of the global concentration tensor associated to each phase given by:

$$
A_{M n K l}^{k}=a_{M n P v}^{k}\left[\sum_{k=1}^{n}\left(\frac{f_{k}}{f_{I}}\left(I_{K I R s}+\frac{1}{V_{k}} T_{i J K l}^{l k}\left(E^{0}\right) \Delta E_{i J R s}^{(k / 0)}\right) a_{R s P v}^{k}\right)\right]
$$

where $a_{M n P v}^{k}$ is the local localization tensor and its expression is given by:

$$
\begin{aligned}
& a_{M n K l}^{k}=\left(\sum_{i=1}^{k-1} f_{i} W_{M n R s}^{(i / k)} a_{R s K l}^{i}\right) / \sum_{i=1}^{k-1} f_{i} \\
& \text { and } W_{M n R s}^{(i / k)}=I_{M n R s}+T_{i J M n}^{k}\left(E^{k}\right) \Delta E_{i J R s}^{(i / k)}
\end{aligned}
$$

in which $I$ is the identity tensors and the explicit formulations of tensor $T_{i J M n}^{l k}$ is given in [6]. The computation of the localization tensors requires the interaction tensors $T^{K}$ and $T_{i J M n}^{I k}$ which have to be first numerically computed.

\subsection{Functionally graded interphase}

Let us consider a three phase composite in which the interphase between the matrix and the reinforcements varies continuously. Note that, phase 1 is reinforcement, phase 3 is matrix, both having homogeneous material properties. The phase 2 is the interphase with spatially varying properties. The reinforcements are perfectly aligned and have ellipsoidal shape with the dimensions $a_{1}, b_{1}, c_{1}$ and $a_{2}, b_{2}, c_{2}$. The two ellipsoids are assumed here to be coaxial with similar shape $\left(\frac{a_{1}}{a_{2}}=\frac{b_{1}}{b_{2}}=\frac{c_{1}}{c_{2}}=\gamma\right)$. The volume fraction of the matrix is $f_{3}$ and the volume fraction of the inclusions and interphases are obtained by $f_{1}=\left(1-f_{3}\right) \gamma^{3}$ and $f_{2}=\left(1-f_{3}\right)\left(1-\gamma^{3}\right)$.

The magnetoelectroelastic moduli $E^{2}(r)$ of the interphase is taken as a radial function and its expression is given by

$$
E^{2}(r)=\left(\alpha E^{1}-E^{3}\right)\left(\frac{1-r}{1-\gamma}\right)^{n}+E^{3}
$$

with $0 \leq \alpha \leq 1, \quad n=2,3 \ldots$ and $\mathrm{r}$ represents the normalized radial distance from the inner surface of the interphase ranging from $\gamma$ to 1 . This model allows accounting for the graded interphase effect. For the considered coating behavior there are two limiting cases: one is when $\gamma$ approaches zero and then phase one disappears, the other is when $\gamma$ approaches 1 which corresponds to no interphase between the matrix and the inclusion.

The mathematical developments presented in this section and in reference [6] are applied to predict the 
effective properties of the composite described in this section. Various micromechanical models are elaborated.

\subsection{Micromechanical approaches and effective properties}

\subsubsection{Self Consistent approach}

The Self Consistent approach takes into account the interaction between the matrix and the multi-coated reinforcements. In the Self Consistent approach the composite is considered as a multi-coated inclusion embedded in a Matrix which takes the properties of the whole composite. In this case, the expression of the effective moduli of magnetoelectroelastic composites with multi-coated reinforcements is written as follow:

$E_{i J K l}^{e f f}=E_{i J K l}^{M}+\sum_{k=1}^{n} f^{k}\left(E_{i J M n}^{k}-E_{i J M n}^{M}\right) A_{M n K l}^{k}$

From this equation it is seen that the determination of the effective moduli of magnetoelectroelastic composites with multi-coated reinforcements requires only the evaluation of the localization tensors $A^{k}$. For the considered composites, the expression of the Self Consistent concentration tensor $A^{k}$ is given by:

$A_{M n K l}^{k}=a_{M n P_{v}}^{k}\left[\sum_{k=1}^{n}\left(\frac{f_{k}}{f_{I}}\left(I_{K I R s}+\frac{1}{V_{k}} T_{i J K l}^{I k}\left(E^{\text {eff }}\right) \Delta E_{i J R s}^{(k / \text { eff })}\right) a_{R s P_{v}}^{k}\right)\right]^{-1}$

in which $\Delta E_{i J M n}^{(k / e f f)}=E_{i J M n}^{k}-E_{i J M n}^{e f f}$

Note that $A^{k}$ depends on the tensor $E^{e f f}$ which is not yet known. An iterative procedure is used for numerical solution.

\subsubsection{Incremental Self Consistent approach}

The classical Self Consistent approach badly estimates the effective behavior of the composites at high volume fraction of the reinforcements and is limited for very low concentration for composites materials containing voids or composites with high contrast between the matrix and the inclusions. An improvement of the classical Self Consistent by incremental way was developed for two phase piezo composites materials by Fakir and Azrar (2010) and recently extended by Bakkali et al. (2011) for $\mathrm{N}$-phase magnetoelectroelastic composite materials. In this paper, the Incremental Self Consistent method is extended to case of magnetoelectroelastic composites with multi-coated inclusions. The ISCM constructed the effective behavior of composites by replacing a finite increment of the volume fraction of the homogeneities in a certain effective medium, and for each increment the SCM is applied to calculate the effective magnetoelectroelastic properties of the composites materials. The expression of the finite volume fraction to be injected in each step is given by [6]:

$$
\Delta f_{\mathrm{i}}^{\mathrm{k}}=\frac{\Delta f_{k}}{1-\sum_{k=1}^{n}(i-1) \Delta f_{k}}
$$

in which $\Delta f_{k}=\frac{f_{k}}{S}$ is considered as the partial concentration of the considered phase and $S$ is the number of steps. The overall properties of the composites given by the ICSM depend on the number of steps and it is written as follow:

$$
E_{i J K l}^{e f f(i)}=E_{i J K l}^{e f f(i-1)}+\sum_{k=1}^{n} \Delta f_{\mathrm{i}}^{\mathrm{k}}\left(E_{i J M n}^{k}-E_{i J M n}^{e f f(i-1)}\right) A_{M n K l}^{k}
$$

with $E^{e f f(0)}=E^{M}$

This model has been well tested for piezoelectric and magnetoelectroelastic heterogeneous materials and improved the Self Consistent predictions [1,6].

\subsubsection{Mori-Tanaka mean field approach}

The Mori-Tanaka mean field approach takes into account the effect of other multi-coated inhomogeneities by considering a finite concentration of a multi-coated inclusion embedded in an infinite matrix. The expression of the Mori Tanaka concentration tensor $A^{k}$ is given by:

$$
A_{M n K l}^{k}=a_{M n P_{v}}^{k}\left[\sum_{k=1}^{n}\left(\frac{f_{k}}{f_{I}}\left(I_{K I R s}+\frac{f_{M}}{V_{k}} T_{i J K l}^{I k}\left(E^{M}\right) \Delta E_{i J R s}^{(k / M)}\right) a_{R s P_{v}}^{k}\right)\right]^{-1}
$$

in which $\Delta E_{i J M n}^{(k / M)}=E_{i J M n}^{k}-E_{i J M n}^{M}$ and $f_{M}=1-f_{I}$ the volume fraction of the matrix.

These models are used for the prediction of effective properties of magnetoelectroelastic heterogeneous materials with multi-coated inclusions and functionally graded interphases. Various numerical results are obtained and only two of them are presented here.

\section{Numerical results}

\subsection{Multi-coated magnetoelectroelastic composites}

In this subsection, a four-phase magnetoelectroelastic composite consisting of piezoelectric inclusions surrounded by an elastic and a piezoelectric layers respectively embedded in a piezomagnetic matrix is considered.

In figure 2 , the electromagnetic coefficient $\alpha_{33}$ is predicted, for magnetoelectroelastic fibrous composite consisting of $\mathrm{BaTiO}_{3}$ inclusions surrounded by Glass and PZT-5H coatings respectively embedded in $\mathrm{CoFe}_{2} \mathrm{O}_{4}$ matrix, by using Mori-Tanaka model as function of the volume fraction of the multi-coated inclusion and the coating thickness of interphases $\left(1-\gamma_{1}\right)$ and $\left(1-\gamma_{2}\right)$. It can be shown that the effective modulus $\alpha_{33}$ is strongly influenced by the thickness of interphases $\left(1-\gamma_{1}\right)$ and (1$\left.\gamma_{2}\right)$. It is also seen that the electromagnetic modulus $\alpha_{33}$ decreases when the thickness of the coatings increases. Note that this coupling coefficient is created by the 
interaction between the piezo and magnetic phases. This coupling effect is of big interest for the multi functionality of these new materials.

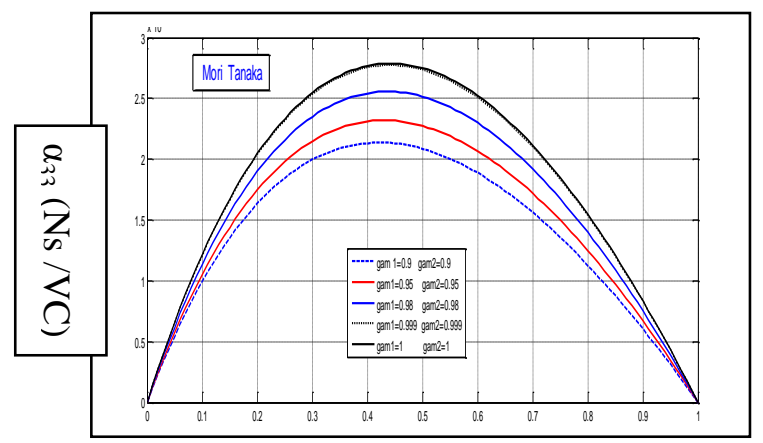

Volume fraction of the multi-coated inclusion

Fig. 2. Effective modulus $\alpha_{33}$, of magnetoelectroelastic fibrous composites consisting of $\mathrm{BaTiO}_{3}$ inclusions surrounded by Glass and PZT-5H coatings embedded in $\mathrm{CoFe}_{2} \mathrm{O}_{4}$ matrix, as a function of the volume fraction of the multi-coated inclusion and the thickness of interphases $\left(1-\gamma_{1}\right)$ and $\left(1-\gamma_{2}\right)$.

\subsection{Magnetoelectroelastic composites with functionally graded interphase}

In this study, a three-phase magnetoelectroelastic composite consisting of piezoelectric inclusions, piezomagnetic matrix with interphase. The properties of interphases vary continuously between the matrix and inclusion. The parameters $(n=2)$ and $(\alpha=1)$, in the mathematical model (6) describing the variation of the properties in the interphase, are chosen. In figure 3 , the electromagnetic coefficient $\alpha_{33}$ is predicted, for magnetoelectroelastic fibrous composites consisting of piezomagnetic matrix $\mathrm{CoFe}_{2} \mathrm{O}_{4}$ and piezoelectric inclusions $\mathrm{BaTiO}_{3}$ with functionally graded interphase, by using the Mori Tanaka's model as function of the volume fraction of the coated inclusion and the thickness of the interphase $\gamma$. It is shown that $\alpha_{33}$ is strongly influenced by the thickness of the interphase $(1-\gamma)$ and that $\alpha_{33}$ decreases as the thickness of the interphase increases.

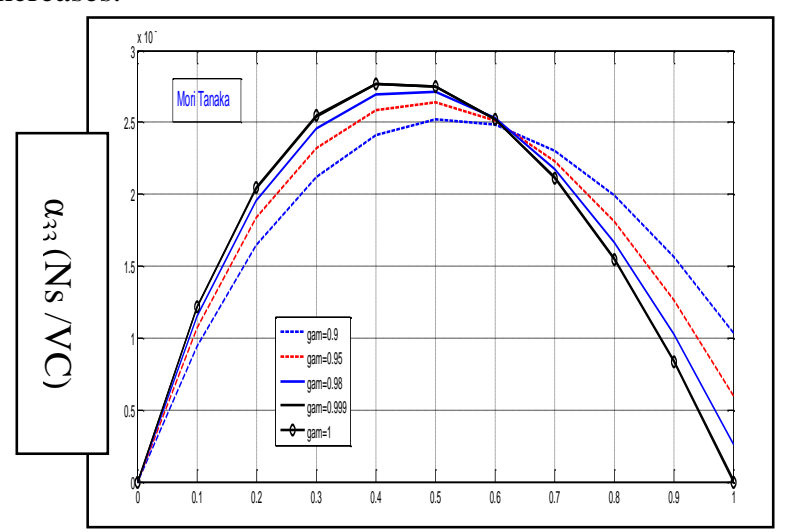

Volume fraction of the coated inclusion

Fig.3. Effective modulus $\alpha_{33}$, of magnetoelectroelastic fibrous composites consisting of piezomagnetic matrix $\mathrm{CoFe}_{2} \mathrm{O}_{4}$ and piezoelectric inclusions $\mathrm{BaTiO}_{3}$ with functionally graded interphase as a function of the volume fraction of the coated inclusion and the thickness of the interphase $(1-\gamma)$.

\section{Conclusion}

The micromechanical modeling developed in this paper allows predicting the effective properties of magnetoelectroelastic composites with multi-coated inclusions and functionally graded interphases. Two kinds of magnetoelectroelastic composite materials have been investigated. The first one is a four-phase composite based on elastic inclusions surrounded by two different piezoelectric layers that is embedded in a piezomagnetic matrix. The second one is a three-phase composite constituted by piezoelectric inclusions, piezomagnetic matrix and a continuously varying interphase between the matrix and inclusion. The effective properties are presented in term of the volume fraction, thickness and graded interphase parameters.

\section{References}

1. N. Fakri, L. Azrar, "Thermal and electro-elastic behaviour of piezo-composites and inhomogeneous piezoelectric materials with voids" Journal of Intelligent Materials Systems and Structures, vol: 21, $\mathrm{N}^{\circ} 2$, pp 161-174, (2010).

2. X. Feng, D. Fang, K. Hwang, "Closed-form solution for piezomagnetic inhomogeneities embedded in a non-piezomagnetic matrix" European Journal of Mechanics A/Solids, Vol. 23, pp. 1007-1019, (2004).

3. Z. K. Zhang, A. K. Soh, "Micromechanics predictions of the effective moduli of magnetoelectroelastic composite materials" European. J. of Mechanics A/Solids, Vol. 24, pp.1054-1067, (2005).

4. S. Srinivas, J. Y. Li, Y. C. Zhou, A. K. Soh, " The effective magnetoelectroelastic moduli of matrixbased multiferroic composites" Journal of Applied Physics, Vol. 99, 043905, (2006).

5. J. Lee, J. G. Boyd, DC. Lagoudas, (2005), "Effective properties of three-phase electro-magneto-elastic composites" Int. J. Engineering Science, Vol. 43, pp. 790-825.

6. A. Bakkali, L. Azrar, N. Fakri, "Modeling of effective properties of multiphase magnetoelectroelastic heterogeneous materials" Journal Computers, Materials \& Continua, Vol. 23, pp. 201-232, (2011).

7. M. Cherkaoui, H. Sabar, M. Berveiller, "Micromechanical approach of the coated inclusion problem and applications to composites materials” $\mathrm{J}$. Eng. Mater. Technol. 116, 247-278, (1994).

8. S. Berbenni, M. Cherkaoui, "Homogenization of multicoated inclusion-reinforced linear elastic composites with eigenstrains" Philosophical Magazine 90, pp. 3003-3026, (2010).

\section{Acknowledgements}

The authors would like to acknowledge the financial support provided by the CNRST in Morocco for the Materials World Network research project $N^{\circ}$ 96/2010 and from the DSR research project at King Abdulaziz University in Jeddah, Saudi Arabia. 